\title{
Comorbid Bipolar Disorder and Obsessive-Compulsive Disorder: An Old Debate Renewed
}

\author{
Andrea Amerio ${ }^{1,2} \bowtie$, Matteo Tonna ${ }^{3}$, Anna Odone ${ }^{4}$, and S. Nassir Ghaemi ${ }^{2,5}$ \\ ${ }^{1}$ Department of Clinical and Experimental Medicine, University of Parma, Parma, Italy \\ ${ }^{2}$ Mood Disorders Program, Tufts Medical Center, Boston, MA, USA \\ ${ }^{3}$ Department of Mental Health, Local Health Service, Parma, Italy \\ ${ }^{4}$ Department of Biomedical, Biotechnological and Translational Sciences, Unit of Public Health, University of Parma, Parma, Italy \\ ${ }^{5}$ Tufts University Medical School, Department of Psychiatry and Pharmacology, Boston, MA, USA
}

\section{To the Editor,}

Apparent comorbidity between bipolar disorder (BD) and anxiety disorders is a common condition in psychiatry with higher prevalence rates for generalised anxiety disorder, social phobia, panic disorder. ${ }^{1}$

Overlapping clinical criteria for many diagnoses, especially mood and anxiety disorders, produced by the Diagnostic and Statistical Manual of Mental Disorders (DSM) might lay behind these high prevalence rates. ${ }^{2}$ Using DSM, it is unclear whether concomitant diagnoses actually reflect the presence of distinct clinical entities or refer to multiple manifestations of a single clinical entity.

With regard to anxiety disorders, one of the most difficult additional psychiatric diagnosis to manage in $\mathrm{BD}$ patients is obsessive-compulsive disorder (OCD)., ${ }^{3,4}$ In our recent metaanalysis, the pooled prevalence of $\mathrm{OCD}$ in $\mathrm{BD}$ was $17.0 \%$, which was comparable to the results reported by the pooled prevalence of $\mathrm{BD}$ in $\mathrm{OCD}(18.35 \%){ }^{5}$ In line with previous studies, ${ }^{6} \mathrm{OCD}$ prevalence rate was higher among $\mathrm{BD}$ children and adolescents (24.2\%, compared to $13.5 \%$ in adults).

The co-occurrence of symptoms of $\mathrm{BD}$ and $\mathrm{OCD}$ was noted 150 years ago by Morel, ${ }^{7}$ but the topic is insufficiently studied and the relationship between $\mathrm{BD}$ and $\mathrm{OCD}$ remains unclear.

In agreement with Kraepelin's thought a psychiatric diagnosis is best established by its longitudinal course of illness, the evidence so far supports the view that, especially among pa-

Received: October 7, 2015 Revised: November 22, 2015

Accepted: November 22, 2015 Available online: March 23, 2016

$\triangle$ Correspondence: Andrea Amerio, MD

Department of Clinical and Experimental Medicine, University of Parma, c/o Ospedale Maggiore, Viale A. Gramsci 14, Parma 43126, Italy

Tel: +390521 903594, Fax: +390521347047

E-mail: andrea.amerio@studenti.unipr.it

(a) This is an Open Access article distributed under the terms of the Creative Commons Attribution Non-Commercial License (http://creativecommons.org/licenses/bync/3.0) which permits unrestricted non-commercial use, distribution, and reproduction in any medium, provided the original work is properly cited. tients with a primary diagnosis of $\mathrm{BD}$, the majority of comorbid OCD cases appeared to be related to mood episodes. ${ }^{8} \mathrm{OC}$ symptoms in comorbid patients appeared more often-and sometimes exclusively-during depressive episodes, and comorbid BD and OCD cycled together, with OC symptoms often remitting during manic/hypomanic episodes.

Therefore, only a substantial minority of comorbid BDOCD may represent "true" OCD independent of $\mathrm{BD}$ with $\mathrm{OC}$ symptoms that improve or worsen during mood episodes without being related to these.

These findings are in line with Feinstein's definition of $\mathrm{co}^{-}$ morbidity, as "any distinct additional entity that has existed or may occur during the clinical course of a patient who has the index disease under study". "True" OCD comorbid with $\mathrm{BD}$ would represent the random co-occurrence of two independent diseases, estimated by multiplication of prevalence rates, which are about $1 \%$ for each condition, and producing a very low expected true comorbidity of $0.1 \%$ in the general population.

The treatment of $\mathrm{BD}-\mathrm{OCD}$ patients remains a great challenge, since the gold standard for one disease (serotonin reuptake inhibitors for OCD) can worsen the other (antidepressants can cause mania and/or more mood episodes in BD).

The clinical features of comorbid BD-OCD patients would explain why $\mathrm{OCD}$ and $\mathrm{BD}$ symptoms respond to adequate mood stabilizer treatment. ${ }^{3}$ Only in a minority of comorbid patients with persistent $\mathrm{OCD}$, despite improvement in mood episodes, addition of low doses of antidepressants could be considered while strictly monitoring emerging symptoms of mania or mixed states. Benefit with antipsychotics was also seen, although a few reports also exist of exacerbation of OC symptoms with neuroleptic agents.

Considering the growing interest over the last decades into shared pathophysiologies across psychiatric disorders, stud- 
ies addressing neurobiological substrates are essential to illuminate pathogenetic mechanisms that underlie comorbid BDOCD.

\section{Acknowledgments}

This research received no specific grant from any funding agency in the public, commercial or not-for-profit sectors.

\section{Conflicts of Interest}

Dr. Amerio, Dr. Tonna, and Dr. Odone report no conflicts of interest. Dr. Ghaemi has provided research consulting to Sunovion and Pfizer, and has obtained a research grant from Takeda Pharmaceuticals. Neither he nor his family hold equity positions in pharmaceutical corporations.

\section{REFERENCES}

1. Boylan KR, Bieling PJ, Marriott M, Begin H, Young LT, MacQueen GM. Impact of comorbid anxiety disorders on outcome in a cohort of patients with bipolar disorder. J Clin Psychiatry 2004;65:1106-1113.

2. Maj M. "Psychiatric comorbidity": an artefact of current diagnostic systems? Br J Psychiatry 2005;186:182-184.

3. Amerio A, Odone A, Marchesi C, Ghaemi SN. Treatment of comorbid bipolar disorder and obsessive-compulsive disorder: a systematic review. J Affect Disord 2014;166:258-263.

4. Amerio A, Odone A, Marchesi C, Ghaemi SN. Do antidepressant-induced manic episodes in obsessive-compulsive disorder patients represent the clinical expression of an underlying bipolarity? Aust N Z J Psychiatry 2014;48:957.

5. Amerio A, Stubbs B, Odone A, Tonna M, Marchesi C, Ghaemi SN. The prevalence and predictors of comorbid bipolar disorder and obsessivecompulsive disorder: a systematic review and meta-analysis. J Affect Disord 2015;186:99-109.

6. Sala R, Axelson DA, Castro-Fornieles J, Goldstein TR, Goldstein BI, Ha $\mathrm{W}$, et al. Factors associated with the persistence and onset of new anxiety disorders in youth with bipolar spectrum disorders. J Clin Psychiatry 2012;73:87-94.

7. Morel BA. Traité des maladies mentales. Second Edition. Paris: Masson; 1860

8. Amerio A, Odone A, Liapis CC, Ghaemi SN. Diagnostic validity of comorbid bipolar disorder and obsessive-compulsive disorder: a systematic review. Acta Psychiatr Scand 2014;129:343-358.

9. Ekman CJ, Lind J, Rydén E, Ingvar M, Landén M. Manic episodes are associated with grey matter volume reduction - a voxel-based morphometry brain analysis. Acta Psychiatr Scand 2010;122:507-515. 